\title{
Physics-driven Locomotion Planning Method for Multilegged Robots
}

\author{
Fei Zhang, Yang Yu \\ Beihang University \\ 37 Xueyuan Road, Haidian District, Beijing, China \\ yu.yang@buaa.edu.cn
}

\begin{abstract}
Deformable robots have an excellent performance in unstructured terrain, because of the highly adaptive configuration. However, most current locomotion planning methods for deformable robots require elaborate manual intervention, so autonomy is urgently demanded. We propose a physics-driven locomotion planning method for multilegged robots. Using an artificial dynamic model, this method can produce the control command automatically once given the desired path. The artificial dynamic model includes only a few parameters with definite physical meanings. This method is evaluated on a 32-legged spherical robot using MSC ADAMS (Automated Dynamic Analysis of Mechanical Systems). The results show that our method is capable of generating a feasible locomotion control scheme for multilegged robots to track smooth paths.
\end{abstract}

Keywords: Automatic locomotion planning, Terrain adaptive, Multilegged robot, Artificial dynamic model

\section{Introduction}

Unstructured complex terrain is a common problem faced by mobile robots. Such scenarios include planet exploration, disaster relief, reconnaissance. The paradigms of mobile robots are wheeled robots, multi-legged robots, and tracked robots[1]. However, most of them have limits in locomotion on complex terrains due to the low flexibility in structure arrangement.

Deformable robot or shape-changing robot is a promising approach for this problem, owing to its ability to reshape itself to adapt to the terrain surface. Most deformable robots have highly symmetrical structures with many degrees of freedom, which contribute to terrain adaptability and omnidirectional locomotion[2]. Deformable robots can be divided into twodimension robots and three-dimension robots. Two-dimension deformable robots include circular robots, polygonal robots [3]; three-dimension deformable robots include tensegrity robots, edge skeleton robots, and radial skeleton robots[2].

The locomotion of deformable robots follows a similar principle: move the center of gravity forward through continuous shape changing[4]. During this deformation process, contact forces and gravity will cause the robot to roll and move forward. Locomotion planning methods can be divided into manual discrete planning and automatic planning. Manual discrete locomotion planning method can tap the robot's motion potential as much as possible, but it is too complicated. Automatic locomotion planning can be produced by the center pattern generator (CPG)[5], certain periodic functions[6], or inverse kinematics[2]. However, these methods are hard to apply to different terrains or require a lot of extra information. Thus, a locomotion planning method, which can generate control commands automatically for different tasks, is highly demanded.

In this paper, we propose a physics-driven locomotion planning method, which can give a feasible control scheme for the multilegged spherical robot to follow a given path on complex terrains. In this method, we virtualize a dynamic model for the multilegged robot. The control command can be obtained from this artificial dynamic model, which has only a few parameters with clear physical meanings. We implement this method in some typical tasks and evaluate its performance through dynamic simulation in ADAMS.

\section{Physics Driven Locomotion Planning 2.1. 32-Legged Spherical Robot}

A 32-legged spherical robot, as shown in Fig.1, is used to test this locomotion planning method. The orientation of 32 legs is determined following the structure of the truncated icosahedron, which has 32 faces and 60 vertices. The line on which each leg is located passes through the center of a face on the truncated icosahedron. A telescopic actuator is equipped on each leg, which is connected to the central spherical. Assuming that the mass of the robot is evenly distributed, we can change 
the length of legs to change the shape of the robot through these telescopic actuators. Thus, we can make the robot move forward following a specified path on different terrains by continuously changing its shape.

In this $3 \mathrm{D}$ model, the central spherical body with a radius of $0.1 \mathrm{~m}$ is a mounting base for other components. The length of the leg is defined as the distance from the robot's center to the end of the telescopic actuator. Here, the length of each leg can vary between $0.35 \mathrm{~m}$ and $0.5 \mathrm{~m}$, i.e., the extension ratio is about $42.857 \%$.
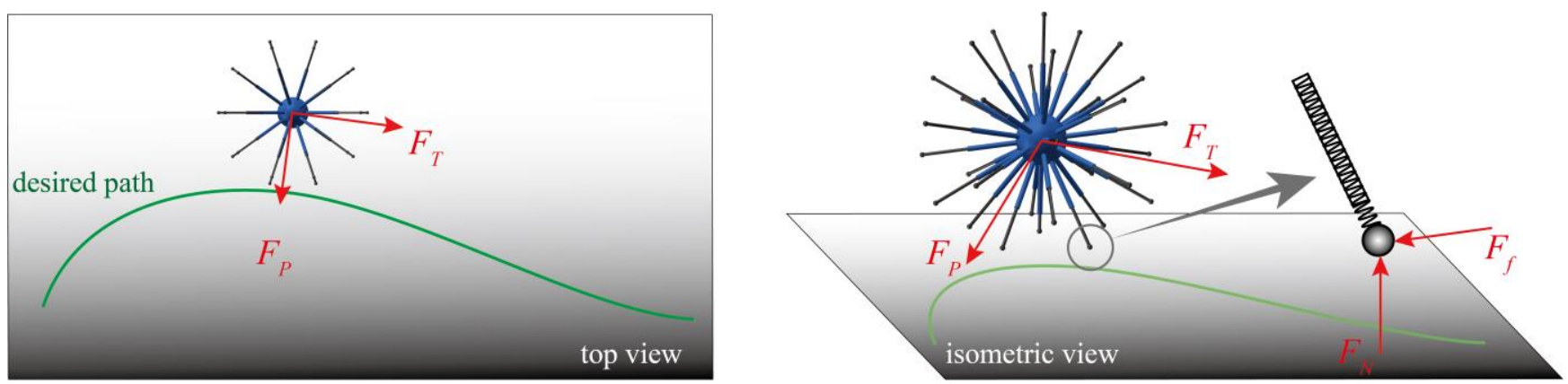

Fig. 1: 32-legged spherical robot in the artificial dynamic model.

\subsection{Physics Driven Locomotion Planning Method}

The physics-driven locomotion planning method can give guidance to the leg length control for a specific path, using an artificial dynamic model. This method is inspired by the artificial potential field method (APF), which is an efficient approach to collision-free motion planning. In the APF method, the point that represents the robot in configuration space moves under the influence of a potential field that combines attraction to the goal and repulsion from obstacles. The APF method will generate generalized force, acceleration, or velocity of the robot[1].

In the artificial dynamic model, not only the force field but also the mechanical structure is artificial. To get the control command for a given path, we set up an artificial dynamic model as follows:

a) The prismatic joint between the leg and the body is virtualized as a spring with damping in a sleeve.

b) The ground does not deform in the contacting process and only produces geometric constraints.

c) There will be an artificial thrust force along the tangential direction of the expected path.

d) There will be an artificial penalty force to pull the robot back when the robot deviates from the expected path.

All the parameters used have definite physical meaning, so we call it physics-driven locomotion planning method. In this artificial model, the robot will roll forward following the expected path due to the artificial forces. The length of each leg in this virtualized dynamic model is extracted as the control input of the real physical model. By adjusting the dynamic parameters in the artificial model, we can ensure that at least three legs are in contact with the ground at the same time. It is expected that the motion obtained in the artificial dynamic model can be reproduced in the physical prototype if the relative motion in the contact is small enough.

In this artificial dynamic model, the robot is treated as a single rigid body, and the length of each supporting leg is calculated by the contact position with the ground. In this artificial model, robot mass equals $10 \mathrm{~kg}$; stiffness and damping coefficient of the leg's spring equal $500 \mathrm{~N} / \mathrm{m}$ and $50 \mathrm{~N} \cdot \mathrm{m} / \mathrm{s}$ respctively; ground friction coefficient is 0.5 . Three coordinates in the Cartesian coordinate system are used to present the position of the center of mass of this robot. Quaternions instead of Euler angles are used to describe the robot's orientation to avoid the singularity. Thus, the system state variables can be described by

$$
\boldsymbol{q}=\left(x, y, z, \lambda_{0}, \lambda_{1}, \lambda_{2}, \lambda_{3}, v_{x}, v_{y}, v_{z}, \omega_{x}, \omega_{y}, \omega_{z}\right)^{\mathrm{T}},
$$

where $(x, y, z),\left(\lambda_{0}, \lambda_{1}, \lambda_{2}, \lambda_{3}\right),\left(v_{x}, v_{y}, v_{z}\right)$ and $\left(\omega_{x}, \omega_{y}, \omega_{z}\right)$ represent the position of the center of mass in the inertial frame, the quaternions, the velocity in the inertial frame and the angular velocity in the body-fixed coordinate system. $\dot{\boldsymbol{q}}$ can be calculated by the Newton-Euler equation. The derivatives of quaternions can be calculated by 


$$
\dot{\boldsymbol{\Lambda}}=\left(\dot{\lambda_{0}}, \dot{\lambda_{1}}, \dot{\lambda_{2}}, \dot{\lambda_{3}}\right)^{\mathrm{T}}=\frac{1}{2}\left[\begin{array}{ccc}
-\lambda_{1} & -\lambda_{2} & -\lambda_{3} \\
\lambda_{0} & -\lambda_{3} & \lambda_{2} \\
\lambda_{3} & \lambda_{0} & -\lambda_{1} \\
-\lambda_{2} & \lambda_{1} & \lambda_{0}
\end{array}\right]\left[\begin{array}{l}
\omega_{1} \\
\omega_{2} \\
\omega_{3}
\end{array}\right]
$$

The forces acting on the robot include gravity $G$, thrust force $F_{T}$, penalty force $F_{P}$, normal contact force $F_{N}$, and friction force $F_{f}$, as shown in Fig.1. The magnitude of $F_{T}$ is constant, and the magnitude of $F_{P}$ is proportional to the square of the deviated distance. The velocity-based friction model is used to calculate the tangential contact force.

\section{Simulations and Results}

To verify this method, we conducted experiments on the 32-legged spherical robot in ADAMS. Then we evaluated the results by comparing the trajectory in the artificial dynamic model for locomotion planning, the trajectory in ADAMS simulation used for verification, and the desired trajectory.

\subsection{Straight Trajectory}

In this task, the robot follows a straight line. As shown in Fig.2, the trajectory in locomotion planning and dynamic simulation can both match the desired path well. The traveling distances in the artificial dynamic model and Adams simulation are $3.28 \mathrm{~m}$ (costing $0.897 \mathrm{~s}$ in MATLAB2020a on AMD 4800U) and $3.40 \mathrm{~m}$ respectively. The mean distance error and maximum distance error in ADAMS simulation relative to the desired path are $2.07 \mathrm{~cm}$ and $4.68 \mathrm{~cm}$ respectively.
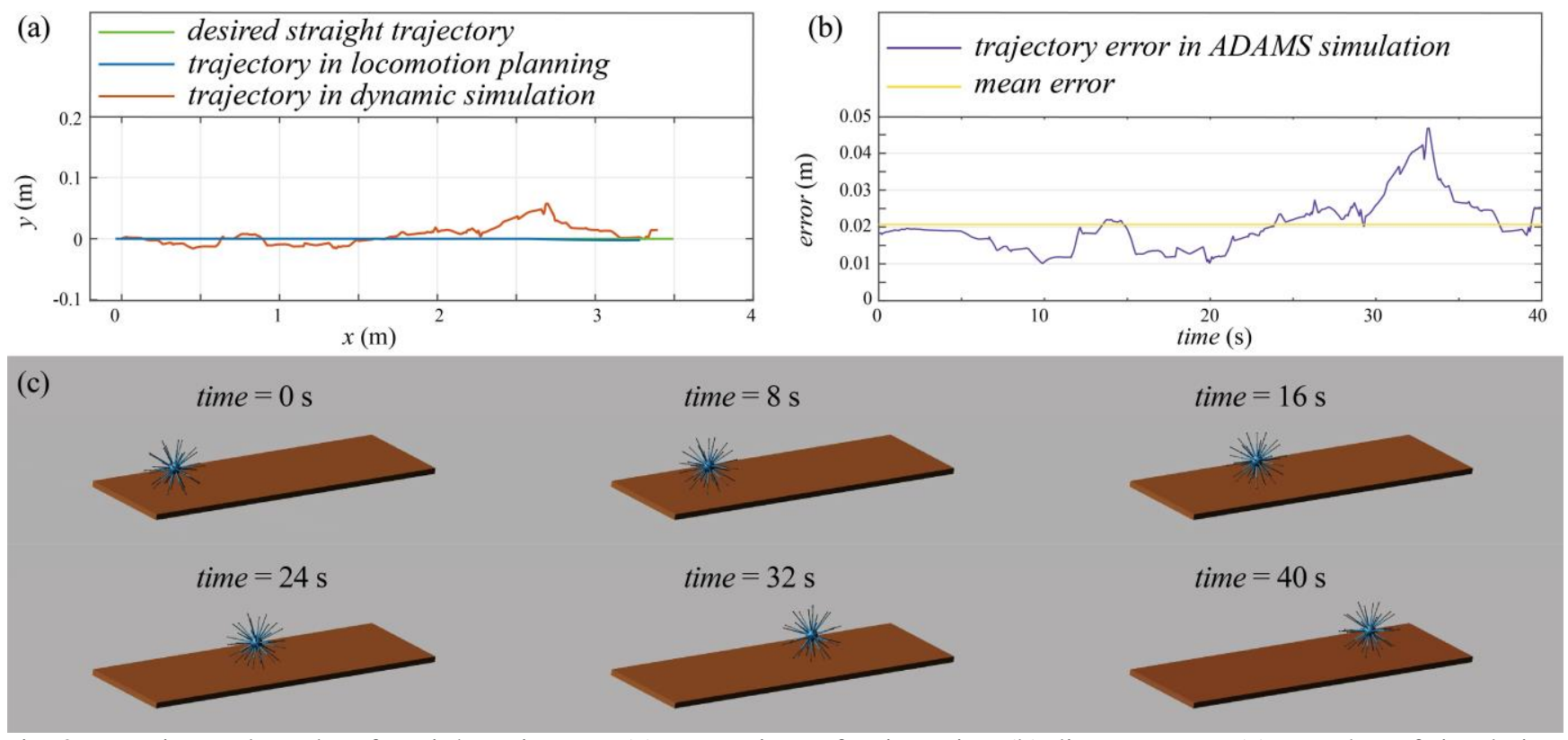

Fig. 2: experimental results of straight trajectory. (a) comparison of trajectories. (b) distance error. (c) snapshot of simulation.

\subsection{Circle Trajectory}

In this experiment, the robot should move around a circle with a radius of $1 \mathrm{~m}$. Experimental results are shown in Fig.3. The path tracking performance in ADAMS simulation is slightly inferior to that in the artificial dynamic model, owning to the difference in artificial thrust force and contact forces. The mean distance error and maximum distance error in ADAMS simulation are $4.64 \mathrm{~cm}$ (costing $0.976 \mathrm{~s}$ in MATLAB2020a on AMD 4800U) and $16.20 \mathrm{~cm}$ respectively. Simulation results indicate that the proposed method performs well in following a given path compared with the previous work[2]. 
(a)

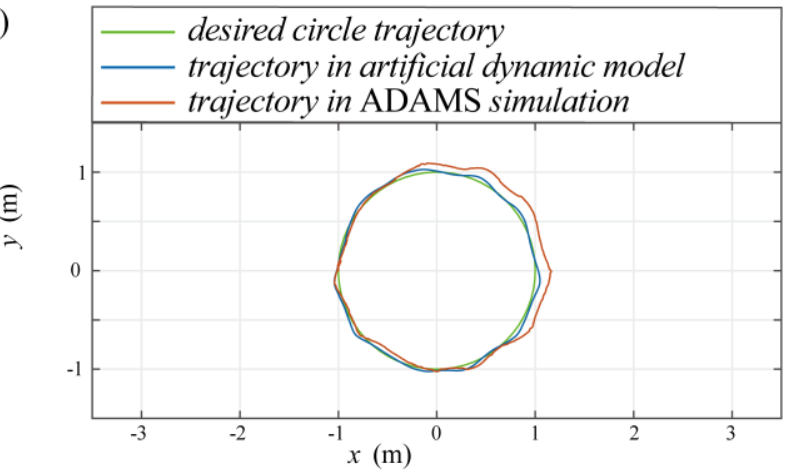

(b)

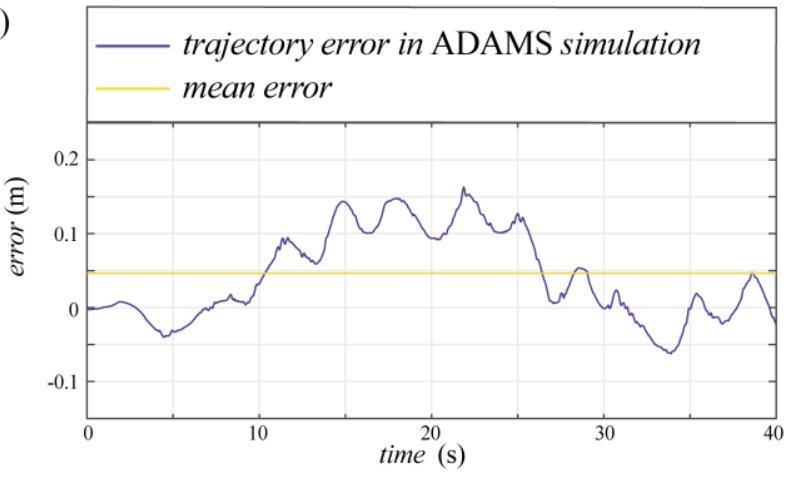

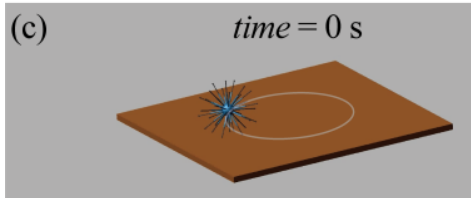

time $=24 \mathrm{~s}$

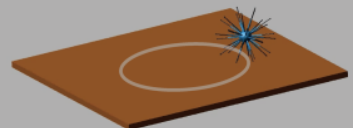

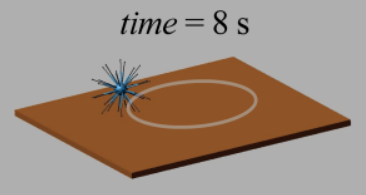

time $=32 \mathrm{~s}$

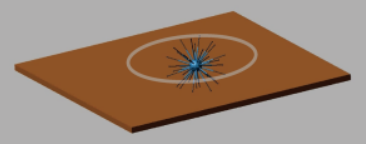

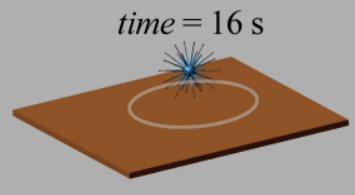

time $=40 \mathrm{~s}$

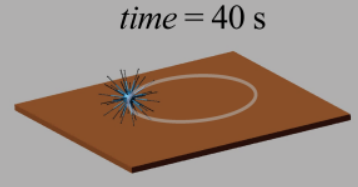

Fig. 3: experimental results of circle trajectory. (a) comparison of trajectories. (b) distance error. (c) snapshot of simulation.

\section{Conclusion}

In this paper, we proposed the physics-driven locomotion planning method for multilegged robots. An artificial dynamic model is used, where all the parameters have definite physical meaning. Numeric simulations show this method can produce feasible locomotion without requiring complex real-world information, which benefits the automatic design of a multi-DOF robotic system. Though the numeric examples employed in this paper are based on flat terrain, we find the method is extensive and is expected to have good performance on unstructured terrains and for other types of deformable robots. Our next work will focus on automatic locomotion planning for complex terrains.

\section{Acknowledgements}

This work was supported by the National Natural Science Foundation of China [grant numbers 12022212 and 11702009].

\section{References}

[1] B. Siciliano, L. Sciavicco, L. Villani, and G. Oriolo, Robotics: modelling, planning and control. Springer Science \& Business Media, 2010.

[2] H. Nozaki, Y. Kujirai, R. Niiyama, Y. Kawahara, T. Yonezawa, and J. Nakazawa, "Continuous shape changing locomotion of 32-legged spherical robot," in 2018 IEEE/RSJ International Conference on Intelligent Robots and Systems (IROS), 2018: IEEE, pp. 2721-2726.

[3] F. Zhang, Y. Yu, Q. Wang, X. Zeng, and H. Niu, "A terrain-adaptive robot prototype designed for bumpysurface exploration," Mechanism and Machine Theory, vol. 141, pp. 213-225, 2019.

[4] K. Kim, "Rapid prototyping design and control of tensegrity soft robot for locomotion," in 2014 IEEE International Conference on Robotics and Biomimetics (ROBIO 2014), 2014: IEEE, pp. 7-14.

[5] A. J. Ijspeert, "Central pattern generators for locomotion control in animals and robots: a review," Neural networks, vol. 21, no. 4, pp. 642-653, 2008. 
[6] T. Ohashi, H. Yamada, and S. Hirose, "Loop forming snake-like robot ACM-R7 and its serpenoid oval control," in 2010 IEEE/RSJ International Conference on Intelligent Robots and Systems, 2010: IEEE, pp. 413-418. 